\title{
Avances en investigación de la discapacidad intelectual de origen genético: tratamientos experimentales en el síndrome $X$ frágil
}

\section{Progress in research of intellectual disability caused by genetic disorders: experimental treatments for the fragile $\mathrm{X}$ syndrome}

Palabras clave

Síndrome X frágil, estrés oxidativo, vitaminas, antioxidantes, ensayo

clínico, comportamiento.

\section{Keywords}

Fragile X syndrome, oxidative stress, vitamins, antioxidants, clinical trial, behavior.

\section{Introducción}

Dentro del contexto de la discapacidad intelectual y los trastornos del desarrollo infantil, la investigación se ha interesado en desvelar los mecanismos moleculares alterados por las mutaciones genéticas y en identificar los genes específicos que afectan funciones del sistema nervioso. Desde hace 25 años dedicamos nuestros esfuerzos a diagnosticar y desvelar los posibles mecanismos celulares que conducen a la sintomatología del síndrome X frágil (SXF) y el autismo, entre otros trastornos.

El síndrome $\mathrm{X}$ frágil es la causa más frecuente de discapacidad intelectual hereditaria y autismo (ver Tabla I), afecta aproximadamente a uno de cada $2500-4000$ varones

I. Financiación de la investigación: este trabajo ha sido financiado parcialmente por el Ministerio de Sanidad (TRAi 52, ECIo-I9I, ECI I-434) y la Junta de Andalucía en la Consejería de Salud (PIo9-0507), y la Consejería de Innovación (CTS-546 y PIIo-CTS-05704). Financiado parcialmente con fondos FEDER de desarrollo regional. Yolanda de Diego Otero está integrada en el programa Nicolás Monardes del Servicio Andaluz de Salud.

Agradecimientos: por muchas razones nuestro más sincero agradecimiento va dirigido a las más de I 60 familias que nos han apoyado con su generosidad y voluntad para que podamos desarrollar los ensayos clínicos. No encontraremos suficiente reconocimiento para todos estos pioneros por su apoyo.
Yolanda de Diego Otero

<ydediego@yahoo.es>

Hospital Regional Universitario de Málaga

\section{Lucía Pérez Costillas}

$<$ lpcostillas@gmail.com>

Hospital Regional Universitario de Málaga

\section{Rocío Calvo Medina <rrro@hotmail.com>}

Hospital Regional Universitario de Málaga

Carolina Quintero Navarro <quinteroconsulta@gmail.com>

Hospital Regional Universitario de Málaga

Isabel del Pino Benítez <mima23I4@hotmail.es>

Hospital Regional Universitario de Málaga

\section{Yolanda Casado Martín} <yolcasado@gmail.com>

Hospital Regional Universitario de Málaga

Cristina Nogueira Cobas <crisnoguer4@hotmail.com>

Hospital Regional Universitario de Málaga

Para citar:

De Diego Otero, Y. et al. (20I7):

"Avances en investigación de la discapacidad intelectual de origen genético: tratamientos experimentales en el síndrome X frágil”. Revista Española de Discapacidad, 5 (I): 217-227.

Doi: <https://doi.org/IO.5569/23405I04.05.OI.I2> 
Tabla 1: Características más frecuentes observadas en pacientes

\begin{tabular}{|c|c|}
\hline \multicolumn{2}{|c|}{ Características del síndrome $\mathrm{X}$ frágil } \\
\hline $\begin{array}{l}\text { Faciales } \\
\text { - Cara alargada asimétrica. Frente amplia } \\
\text { - Orejas grandes evertidas de implantación baja } \\
\text { - Mandíbula prominente } \\
\text { - Facies acromegaloides en adultos } \\
\text { - Estrabismo }\end{array}$ & $\begin{array}{l}\text { Cerebrales } \\
\text { - Disminución del vermis cerebelar posterior } \\
\text { - Disminución del girus temporal superior } \\
\text { - Atrofia frontal y parietal mediana } \\
\text { - Aumento de tamaño del cuarto ventrículo } \\
\text { - Aumento de volumen en el hipocampo }\end{array}$ \\
\hline $\begin{array}{l}\text { Boca } \\
\text { - Dentición irregular. Paladar hendido }\end{array}$ & $\begin{array}{l}\text { Cardiacas } \\
\text { - Prolapso de válvula mitral. Dilatación de la aorta } \\
\text { ascendente }\end{array}$ \\
\hline $\begin{array}{l}\text { Articulaciones y esqueleto } \\
\text { - Hiperlaxitud articular en las manos } \\
\text { - Escoliosis. Pies planos y/o zambos }\end{array}$ & $\begin{array}{l}\text { Genitales } \\
\text { - Macroorquidismo postpuberal en varones } \\
\text { - Disfunción ovárica menopausia prematura en } \\
\text { portadoras }\end{array}$ \\
\hline $\begin{array}{l}\text { Musculares } \\
\text { - Hipotonía en la infancia }\end{array}$ & $\begin{array}{l}\text { Oído } \\
\text { - Otitis medias recurrentes y problemas auditivos en } \\
\text { la infancia }\end{array}$ \\
\hline $\begin{array}{l}\text { Psíquicas-neurológicas } \\
\text { - Discapacidad intelectual. Problemas de } \\
\text { aprendizaje } \\
\text { - Comportamiento hiperactivo en la infancia } \\
\text { - Alteraciones del lenguaje. Habla reiterativa } \\
\text { - Estereotipias motoras. Convulsiones y epilepsia } \\
\text { - Rasgos autistas. Contacto ocular escaso } \\
\text { - Agresividad. Ansiedad y depresión }\end{array}$ & $\begin{array}{l}\text { Genéticas } \\
\text { - Dominante ligada al cromosoma X, con penetrancia } \\
\text { incompleta } \\
\text { - Sitio frágil dependiente de folato en la región Xq27.3 } \\
\text { - Expansión de repeticiones CGG }(>52) \text { del gen Fmr-1 } \\
\text { - Metilación de la isla CpG reguladora del gen Fmr-1 } \\
\text { - Ausencia del ARNm transcrito del gen Fmr-1 } \\
\text { - Ausencia o inactividad de la proteína FMRP }\end{array}$ \\
\hline
\end{tabular}

Fuente: elaboración propia.

Figura 1: A) Niño y niña afectados por el síndrome $X$ frágil en la etapa infantil y ellos mismos durante la adolescencia. B) Esquemas de las características faciales

A
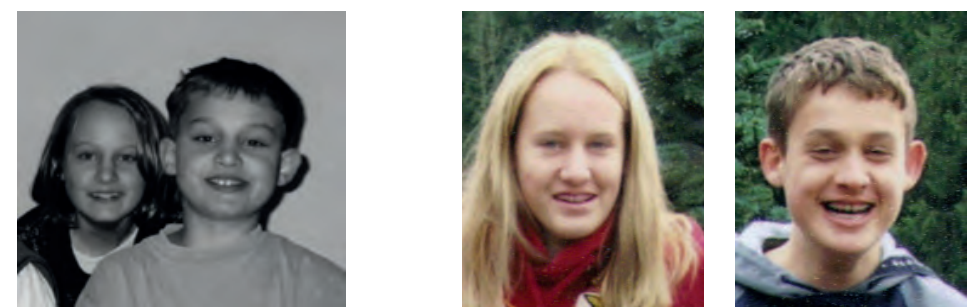

B
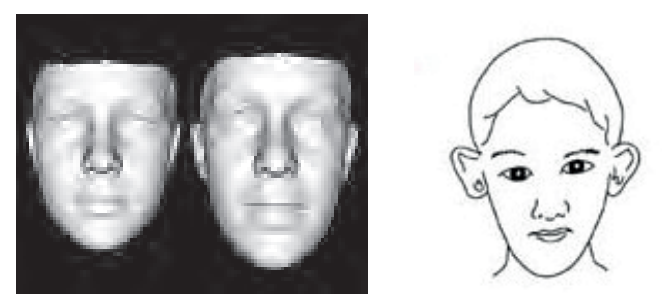

Normal

SXF

Fuente: elaboración propia. 
y una de cada 4000-8000 mujeres y se estima que hay unos I 0.000 afectados en España. El infradiagnóstico detectado en este síndrome hace que solo un 20-30\% de la población afectada esté diagnosticada en la actualidad (FernándezCarvajal et al., 2009: 324).

El síndrome presenta otras características junto a la discapacidad intelectual, como hipotonía en la primera infancia, retraso del desarrollo psicomotor y otitis recurrente en los primeros años de vida, macroorquidismo (tamaño de testículos grandes), displasia del tejido conjuntivo (hiperlaxitud articular, pies planos o prolapso de la válvula mitral), anomalías faciales como cara alargada y orejas grandes y evertidas de implantación baja (ver Figura I), estrabismo y convulsiones. A nivel conductual, entre otras características, están los problemas de comportamiento, la hiperactividad, los rasgos autistas, la agresividad, el retraso en el lenguaje y problemas de aprendizaje (Hagerman y Hagerman, 2002: I).

Estos pacientes necesitarán de un diagnostico precoz y un seguimiento continuado en el sistema sanitario, así como de apoyo en distintos aspectos educativos en la etapa infantojuvenil, atención temprana, integración sensorial y apoyo en el desarrollo psicomotor, logopedia, modificación de conducta, apoyo psicológico en el aula, adaptación curricular, talleres ocupacionales, trabajo adaptado, etc. Estas intervenciones van a permitir un desarrollo de su máximo potencial, así como una integración sociolaboral más adecuada de los afectados (Hagerman y Hagerman, 2002: I).

\section{Características genéticas del síndrome $X$ frágil}

La alteración genética o mutación es una región inestable de ADN en el gen FMR I localizado en la banda Xq23.7 en el cromosoma X. Esta zona aumenta de tamaño por la expansión del número de tripletes CGG (mutación completa con más de 200 CGG), lo que causa la inactivación del gen por metilación del promotor. Se sabe que el tamaño de repeticiones CGG en el gen FMR I es un polimorfismo en la población general normal que puede presentar entre 6 y 54 CGG en la región promotora del gen FMRI, sin que se afecte la inteligencia ni el comportamiento. Nuevos avances apuntan a una posible zona gris que se propone entre 45 y 55 repeticiones que podría ser inestable en familias donde se detecta una pérdida de los tripletes AGG de estabilización que interrumpen la secuencia de CGG cada Io repeticiones y que aparecen siempre en los alelos normales que son estables y no sufren cambios en el número de repeticiones al pasar de una generación a la siguiente (Chiurazzi et al., 2003: 559).

Existe un estado intermedio que se conoce como premutación, cuando el gen FMR I muestra entre 55 y 200 tripletes CGG. Esta alteración en grado de premutación aparece en uno de cada 25 I/470 varones y uno de cada I 5 I/238 mujeres. Los varones portadores de una premutación se lo trasmiten a todas sus hijas que heredan un tamaño similar de premutación con un número parecido de repeticiones CGG al padre, y que normalmente no manifiestan discapacidad intelectual, pero sí podrían manifestar menopausia por fallo ovárico precoz en un $20 \%$ de las mujeres portadoras de premutación menores de 40 años, conocido con el nombre de FXPOI (fragile X-associated primary ovarian insufficiency). También se ha descrito recientemente una nueva sintomatología asociada a la premutación, que ocurre en el $30 \%$ de los portadores mayores de 50 años y se inicia con un parkinsonismo, tremor intencional y ataxia que se conoce con el nombre de FXTAS (fragile X-associated tremor/ataxia síndrome). También hay investigaciones que apoyan la aparición de autismo y de patología psiquiátrica en casos de premutación. Existe un estado que se conoce como mutación completa, cuando el gen FMR I muestra más de 200 tripletes CGG y metilación del promotor. Esta alteración en grado de mutación aparece en uno de cada $2500-$ 4000 varones y uno de cada 4000-6000 mujeres. Cuando la alteración genética inestable, en estado de premutación o mutación completa, se 

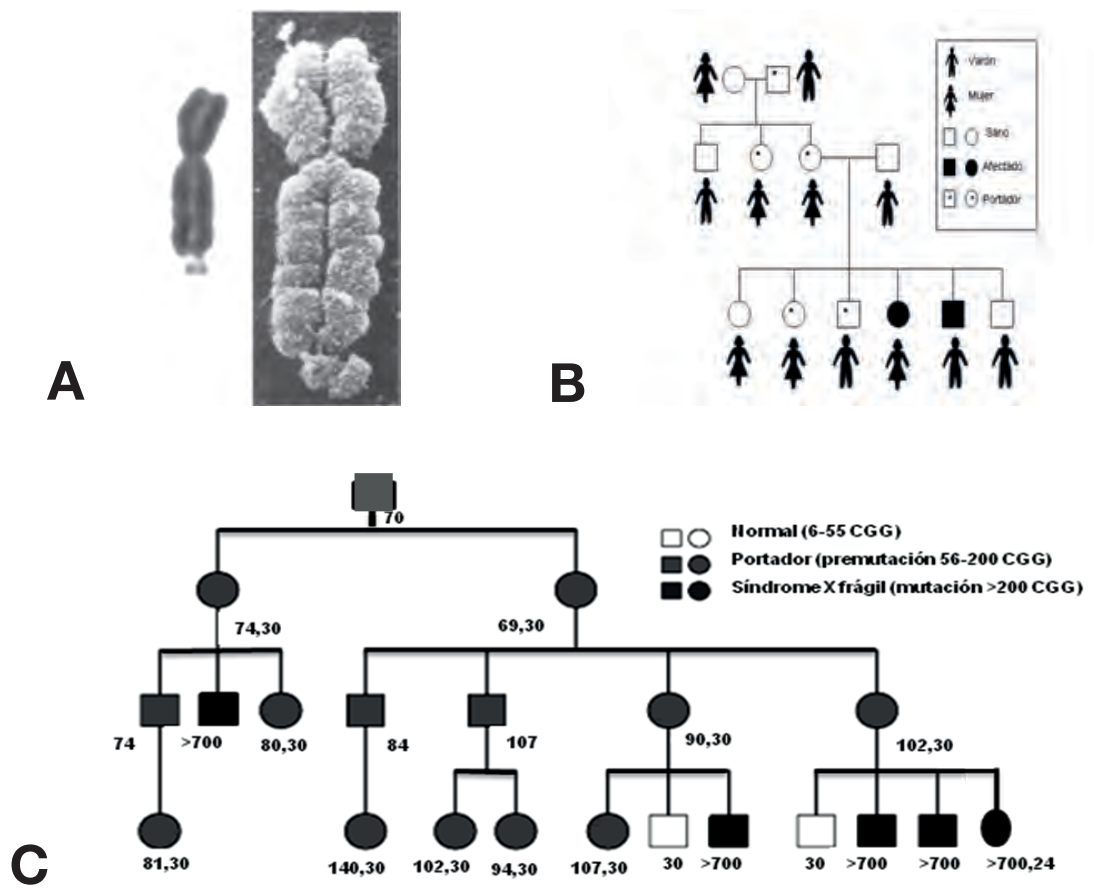

Fuente: elaboración propia.

transmite desde la madre a sus hijos el $50 \%$ de su descendencia tiene riesgo de heredar la mutación y podrían estar afectados en mayor o menor medida por la sintomatología que caracteriza el síndrome (Bourgeois et al., 2009: 852).

El diagnóstico se realiza a través de pruebas genéticas moleculares de $\mathrm{ADN}$, detectando la expansión de los tripletes CGG, junto al nivel de metilación del promotor y las interrupciones AGG que se intercalan entre los tripletes CGG. Se usan principalmente dos técnicas, la del análisis de fragmentos polimórficos (Southern-blot) o por la reacción en cadena de la polimerasa (PCR). El diagnostico precoz es fundamental para que los pacientes puedan seguir un programa de atención temprana, se sabe que tiene efectos muy positivos sobre el desarrollo de las habilidades y capacidades posteriores de los afectados, así como poder desarrollar prevención a través del consejo genético a la familia con riesgo de trasmitir la enfermedad a su descendencia (Grigsby, 20I6: 8I5).

En los centros especializados se propone un equipo multidisciplinar para la atención a los afectados y sus familias, que permita un diagnostico certero, un consejo genético adecuado, así como un apoyo psicológico y asistencia social que les permita conseguir el máximo desarrollo de su potencial y, lo más importante, prevenir nuevos casos a través del uso de los programas establecidos y disponibles en la actualidad, a través de reproducción asistida con diagnostico preimplantacional o donación de ovocitos, o el diagnóstico prenatal de los embarazos de riesgo en familias con herencia del gen FMRI inestable por expansión de tripletes CGG (Hagerman y Hagerman, 2002: I). 


\section{Avances en investigación y tratamientos experimentales}

El conocimiento adquirido gracias a los avances en genética y al estudio de la base fisiopatología del síndrome X frágil permite comenzar a probar nuevas dianas terapéuticas y nuevos tratamientos experimentales. El hecho de que la mutación fuera descrita en I99I, y que el modelo de ratón FmrI-KO se desarrollara muy pronto, han convertido el SXF en modelo en la búsqueda de tratamientos para los trastornos del desarrollo neurológico (Hagerman et al., 20I4).

En la actualidad, los tratamientos farmacológicos utilizados en los pacientes con síndromes que afectan al desarrollo neurológico son únicamente sintomáticos y no modificadores de la enfermedad. No existe aún un enfoque específico que evite la aparición de los síntomas observados en la patología o que intente modular la sintomatología a largo plazo. Esto provoca que no haya unas guías claras de tratamiento y que cada paciente reciba un fármaco u otro dependiendo del síntoma a tratar, con escasa evidencia científica y generalmente baja eficacia, y asociando además muchos efectos adversos y comorbilidad a los pacientes.

Existen bastantes ensayos preclínicos sobre dianas terapéuticas en animales de experimentación, pero pocos han sido aplicados a humanos y en contados ensayos se ha comprobado eficacia de manera concluyente. Estas investigaciones han tenido diferentes enfoques según sus objetivos, ya sea la restauración de la función del gen FMR I o el tratamiento con fármacos que actúan a diferentes niveles (Saldarriaga et al., 20I4): a nivel de los receptores de neurotransmisores, a nivel de proteínas de señalización intracelular o en las proteínas efectoras sinápticas

Se ha demostrado in vitro la posibilidad de reactivar el gen FMR I desmetilando la mutación completa (Chiurazzi et al., I999). También se estudia cuál es la señal de autorregulación del gen para caracterizarla y ver si se podría influir sobre ella artificialmente, para conseguir transcribir el gen en las células de individuos afectados y obtener así proteína funcional, que pueda evitar la manifestación del síndrome (Tassone et al., 200I).

La proteína FMRP, deficiente o ausente por completo en los pacientes con SXF, está involucrada en la regulación de la estabilidad del RNA, el transporte subcelular y la traducción de mRNAs neurales que codifican proteínas involucradas en el desarrollo de la sinapsis, la plasticidad neuronal y el desarrollo cerebral (Sidorov et al., 20I3; Brown et al., 200I; Chen et al., 2003).

En varias publicaciones se observa la asociación de la falta de la proteína FMRP, cuya ausencia causa el síndrome, con alteraciones de muchos mensajeros de ARN en el sistema nervioso. Esto provoca modificaciones en el número y madurez de las espinas dendríticas que conllevan una alteración en la funcionalidad a nivel de la sinapsis neuronal, con desequilibrio entre la excitación y la inhibición por desaparición o sobreexpresión de receptores de Glutamato y/o de GABA (Gatto y Broadie, 20I0). Estas alteraciones determinarán el fenotipo conductual de los pacientes con SXF. En modelo animal y en estudios postmorten se ha demostrado un exceso de conexiones sinápticas y, además, estas sinapsis no alcanzan un adecuado estado de madurez. Se afectará por tanto la poda sináptica y la selección de conexiones útiles. Ésta puede ser la base de la fisiopatología del fenotipo cognitivo conductual en el SXF (He y PorteraCailliau, 20I3).

Algunos artículos demuestran cómo muchos de los genes cuya trascripción podría estar regulada por FMRP están también alterados en pacientes con trastorno del espectro autista (Iossifov et al., 2012), por lo que terapias eficientes en este síndrome podrían ser útiles también en pacientes con trastorno del espectro autista.

Clásicamente se planteó la terapia con ácido fólico, uno de los pocos fármacos que se pueden recomendar durante la primera infancia ya que 
mejora la conducta y la concentración (Fisch et al., 1988: 393). También algunos compuestos agonistas del receptor alfa-2-adrenérgico, como la clonidina o los inhibidores de la recaptación de serotonina, se emplean con éxito en niños con SXF para tratamiento de la ansiedad o el trastorno comportamental (Berry-Kravis y Potanos, 2004).

Hay antipsicóticos que no son típicamente utilizados en SXF, como es el caso de aripripazol o risperidona, que se pueden utilizar en los casos de autolesiones, hiperactividad severa y autismo relacionados con el SXF (Hagerman, 2006: 63).

El sistema endocannabinoide también parece sufrir alteraciones en el modelo de ratón (Tang y Alger, 20I 5). Mediante aproximaciones farmacológicas y genéticas, se ha observado que el bloqueo de los receptores cannabinoides CBI normaliza el déficit cognitivo, la falta de sensibilización ante estímulos dolorosos y la susceptibilidad a padecer crisis epilépticas que aparecen en el modelo murino de SXF. Estas mejoras a nivel conductual fueron acompañadas de cambios a nivel bioquímico en la vía de señalización intracelular mTOR, clave en el procesamiento cognitivo, así como en la regularización de la densidad y madurez de las espinas dendríticas neuronales. Por otro lado, el bloqueo farmacológico de receptores cannabinoides $\mathrm{CB}_{2}$ normalizó el fenotipo de ansiedad reducida que presentan los ratones con este síndrome (Gomis-Gonzalez et al., 2016).

Algunos estudios demuestran cómo un entorno enriquecido con estímulos mejora la evolución en modelos de ratón SXF. Se sabe que el enriquecimiento aumenta la expresión de los receptores de glutamato AMPA en ambos genotipos (WT y Fmri KO), sugiriendo que las vías de activación glutamatérgicas dependientes de FMRP se encuentran alteradas en el ratón FmrI-KO y pueden ser normalizadas por la estimulación ambiental (Restivo et al., 2005: I I 5 57).

Parece ser que los pacientes con SXF se benefician de terapia ocupacional, de actividades deportivas, integración sensorial y musicoterapia. Es posible conseguir grandes logros de mejoras en el comportamiento, trastornos conductuales y en el autismo de niños con SXF mediante la implantación de técnicas específicas tanto en el ambiente escolar como en el familiar (Dyer-Friedman et al., 2002)

En los últimos años se han publicado una serie de iniciativas terapéuticas experimentales, en base a los hallazgos descritos en investigación básica sobre modelos animales, que indicarían que existen problemas en la neurotransmisión como base fisiopatológica del síndrome. Estos estudios indican un posible exceso de excitación glutamatérgica o disminución en la inhibición gabaérgica.

Experimentos recientes en modelos animales llevan a pensar que el tratamiento con fármacos antagonistas de los receptores del glutamato puede representar un avance significativo en el tratamiento del síndrome. En los últimos estudios publicados sobre tratamientos experimentales desarrollados en modelos animales del síndrome $\mathrm{X}$ frágil se indica que, tanto en el ratón como en el modelo en mosca drosophila, se revierte parcialmente el fenotipo con antagonistas de los receptores del glutamato mGlur 5. Estos hallazgos abren nuevas vías de investigación terapéutica para el síndrome. Otros ensayos han sido propuestos con fármacos antagonistas de receptores de glutamato, como el AFQ०56, para comprobar su efectividad en problemas de comportamiento y aprendizaje en los pacientes. También se ha propuesto un ensayo fase II con el fármaco $\mathrm{RO}_{4917523}$, para comprobar su eficacia en comportamiento y cognición en pacientes. Se ha desestimado la continuación de estos ensayos por falta de eficacia en población adulta y adolescente, pero se está probando en población infantil (ClinicalTrials.gov: NCTo2920892), esperando encontrar mejores resultados que en los estudios en adultos y adolescentes (Fung y Reiss, 2016: 100).

También se ha propuesto una posible disfunción colinérgica en el síndrome X frágil que podría estar implicada en la sintomatología y han descrito que el ensayo con donezepil, un inhibidor de la acetilcolinesterasa, mejora 


\begin{tabular}{|c|c|c|c|c|c|c|c|}
\hline \begin{tabular}{|l|} 
Número \\
de \\
registro \\
del \\
ensayo \\
\end{tabular} & $\begin{array}{l}\text { Tipo/Fase } \\
\text { en la que se } \\
\text { encuentra }\end{array}$ & Fármaco & Población & Diana & Estado & Promotor & Resultados \\
\hline $\begin{array}{l}\text { NCT } \\
02680379\end{array}$ & $\begin{array}{l}\text { Ensayo piloto } \\
\text { Unicéntrico. }\end{array}$ & $\begin{array}{l}\text { Minociclina } \\
\text { Lovastatina }\end{array}$ & $\begin{array}{l}\text { Varones } \\
\text { adolescentes y } \\
\text { adultos }\end{array}$ & $\begin{array}{l}\text { Antibiótico } \\
\text { + Fármaco } \\
\text { anticolesterol }\end{array}$ & $\begin{array}{l}\text { En } \\
\text { reclutamiento }\end{array}$ & $\begin{array}{l}\text { Université de } \\
\text { Sherbrooke. } \\
\text { Qebec. Canada }\end{array}$ & Pendiente \\
\hline \begin{tabular}{|l|} 
NCT \\
03140813 \\
\end{tabular} & $\begin{array}{l}\text { Unicéntrico- } \\
\text { Ensayo Piloto }\end{array}$ & AZD7325 & $\begin{array}{l}\text { Varones } \\
\text { adultos }\end{array}$ & $\begin{array}{l}\text { Modulador del } \\
\text { receptor de } \\
\text { GABA-A }\end{array}$ & Registrado & \begin{tabular}{|l|} 
Children's Hospital \\
Medical Center, \\
Cincinnati. EEUU.
\end{tabular} & Pendiente \\
\hline $\begin{array}{l}\text { NCT } \\
01894958\end{array}$ & $\begin{array}{l}\text { Multicéntrico- } \\
\text { Fase II }\end{array}$ & MNZ-2566 & \begin{tabular}{|l} 
Varones \\
adolescentes y \\
adultos
\end{tabular} & $\begin{array}{l}\text { Antagonista } \\
\text { NMDA }\end{array}$ & Completado & $\begin{array}{l}\text { Neuren } \\
\text { Pharmaceuticals } \\
\text { Limited }\end{array}$ & Pendiente \\
\hline $\begin{array}{l}\text { NCT } \\
01855971\end{array}$ & $\begin{array}{l}\text { Unicéntrico } \\
\text { Ensayo piloto }\end{array}$ & $\begin{array}{l}\text { Flavonol } \\
\text { epigallocatechin } \\
\text { gallate (EGCG). }\end{array}$ & $\begin{array}{l}\text { Varones } \\
\text { adultos }\end{array}$ & $\begin{array}{l}\text { Receptor de } \\
\text { Estrogeno beta } \\
(\text { ER- } \beta)\end{array}$ & Completado & $\begin{array}{l}\text { Hospital del Mar. } \\
\text { Barcelona. }\end{array}$ & Pendiente \\
\hline $\begin{array}{l}\text { NCT } \\
01725152\end{array}$ & $\begin{array}{l}\text { Unicéntrico- } \\
\text { Fase II }\end{array}$ & Ganaxolona & $\begin{array}{l}\text { Adolescentes } \\
\text { y niños }\end{array}$ & $\begin{array}{l}\text { Agonista } \\
\text { GABA-A }\end{array}$ & $\begin{array}{l}\text { En marcha } \\
\text { sin reclutar } \\
\text { pacientes }\end{array}$ & \begin{tabular}{|l|} 
Marinus \\
Pharmaceuticals
\end{tabular} & Pendiente \\
\hline $\begin{array}{l}\text { NTC } \\
01474746\end{array}$ & $\begin{array}{l}\text { Unicéntrico- } \\
\text { Fase II }\end{array}$ & Sertalina & Niños & $\begin{array}{l}\text { Inhibidor de la } \\
\text { recaptación de } \\
\text { serotonina }\end{array}$ & Completado & $\begin{array}{l}\text { Universidad de } \\
\text { California,Davis } \\
\text { (USA) }\end{array}$ & Pendiente \\
\hline $\begin{array}{l}\text { NCT } \\
01911455\end{array}$ & $\begin{array}{l}\text { Multicéntrico- } \\
\text { Fase II }\end{array}$ & Acamprosato & $\begin{array}{l}\text { Adolescentes } \\
\text { y niños }\end{array}$ & $\begin{array}{l}\text { Modulador del } \\
\text { receptor de } \\
\text { NMDA }\end{array}$ & Completado & $\begin{array}{l}\text { Children's Hospital } \\
\text { Medical Center, } \\
\text { Cincinnati }\end{array}$ & Pendiente \\
\hline $\begin{array}{l}\text { NCT } \\
01329770\end{array}$ & $\begin{array}{l}\text { Unicéntrico } \\
\text { Fase II }\end{array}$ & $\begin{array}{l}\text { Vitamina C + } \\
\text { Vitamina E }\end{array}$ & $\begin{array}{l}\text { Adultos, } \\
\text { Adolescentes } \\
\text { y niños }\end{array}$ & Antioxidantes & Finalizado & $\begin{array}{l}\text { Hospital Regional } \\
\text { Universitario de } \\
\text { Málaga }\end{array}$ & $\begin{array}{l}\text { Eficacia a nivel cognitivo } \\
\text { con una potencia del } 65 \\
\% \text { y en lenguaje con un } \\
49 \% \text {. }\end{array}$ \\
\hline $\begin{array}{l}\text { NCT } \\
02942498\end{array}$ & $\begin{array}{l}\text { Unicéntrico } \\
\text { Fase II }\end{array}$ & $\begin{array}{l}\text { Vitamina C + } \\
\text { Vitamina E }\end{array}$ & Niños & Antioxidantes & Finalizado & $\begin{array}{l}\text { Hospital Regional } \\
\text { Universitario de } \\
\text { Málaga }\end{array}$ & Pendiente \\
\hline \begin{tabular}{|l|} 
NCT \\
02126995 \\
\end{tabular} & $\begin{array}{l}\text { Unicéntrico- } \\
\text { Fase II }\end{array}$ & $\begin{array}{l}\text { Metadoxine } \\
\mathrm{MG01 \textrm {Cl }} \\
\end{array}$ & $\begin{array}{l}\text { Adultos y } \\
\text { adolescentes }\end{array}$ & Vitamina 6 & Finalizado & Alcobra Ltd. & Pendiente \\
\hline $\begin{array}{l}\text { NTC } \\
01357239\end{array}$ & $\begin{array}{l}\text { Multicéntrico- } \\
\text { Fase II }\end{array}$ & $\begin{array}{l}\text { Mavaglurant } \\
\text { (AFQ056) }\end{array}$ & $\begin{array}{l}\text { Adolescentes } \\
\text { y niños }\end{array}$ & $\begin{array}{l}\text { Antagonista } \\
\text { mGluR5 }\end{array}$ & Finalizado & $\begin{array}{l}\text { Novartis } \\
\text { (Basilea, Suiza) }\end{array}$ & $\begin{array}{l}\text { Negativos, no } \\
\text { hay mejoría en el } \\
\text { comportamiento } \\
\text { medido con la escala } \\
\text { ABC-C (Jacquemont et } \\
\text { al., 2011) }\end{array}$ \\
\hline \begin{tabular}{|l|} 
NTC \\
01015430 \\
\end{tabular} & $\begin{array}{l}\text { Multicéntrico- } \\
\text { Fase II }\end{array}$ & Basimglurant & Adultos & $\begin{array}{l}\text { Antagonista } \\
\text { mGluR5 }\end{array}$ & Finalizado & Hoffman-La Roche & No hay eficacia \\
\hline $\begin{array}{l}\text { NTC } \\
01013480\end{array}$ & $\begin{array}{l}\text { Multicéntrico- } \\
\text { Fase II }\end{array}$ & $\begin{array}{l}\text { Arbaclofeno } \\
\text { (STX209) }\end{array}$ & $\begin{array}{l}\text { Adultos y } \\
\text { adolescentes }\end{array}$ & $\begin{array}{l}\text { Agonista } \\
\text { GABA-B }\end{array}$ & Finalizado & \begin{tabular}{|l|} 
Seaside \\
Therapeutics, Inc.
\end{tabular} & $\begin{array}{l}\text { Eficacia parcial, cierra } \\
\text { el programa por falta } \\
\text { de financiación por la } \\
\text { empresa. }\end{array}$ \\
\hline $\begin{array}{l}\text { NTC } \\
01053156\end{array}$ & $\begin{array}{l}\text { Unicéntrico- } \\
\text { Fase II }\end{array}$ & Minociclina & $\begin{array}{l}\text { Adolescentes } \\
\text { y niños }\end{array}$ & Antibiótico & Finalizado & $\begin{array}{l}\text { Universidad de } \\
\text { California, Davis } \\
\text { (USA) }\end{array}$ & $\begin{array}{l}\text { Mejoría tanto en el } \\
\text { placebo como en el } \\
\text { tratamiento } \\
\text { (Paribello et al., 2010; } \\
\text { Leigh et al., 2013) } \\
\end{array}$ \\
\hline $\begin{array}{l}\text { NTC } \\
01120626\end{array}$ & $\begin{array}{l}\text { Unicéntrico- } \\
\text { Fase II }\end{array}$ & Donepezil & $\begin{array}{l}\text { Adolescentes } \\
\text { y niños }\end{array}$ & $\begin{array}{l}\text { Droga } \\
\text { colonérgica }\end{array}$ & Finalizado & $\begin{array}{l}\text { Universidad de } \\
\text { Stanford (USA) }\end{array}$ & $\begin{array}{l}\text { No hay mejoría en los } \\
\text { test de inteligencia } \\
\text { y comportamentales } \\
\text { (Sahu et al., 2013) }\end{array}$ \\
\hline
\end{tabular}

Fuente: actualizado del artículo de De Diego Otero et al., 2014. 
significativamente funciones cognitivas y de comportamiento en pacientes afectados. Ensayos de tratamiento han comprobado la efectividad parcial de distintos compuestos, como el litio, que ha demostrado eficacia para mejorar el comportamiento y el aprendizaje (Ligsay y Hagerman, 2016: I 58).

La L-acetilcarnitina demuestra eficacia para reducir la hiperactividad y mejorar la relación social de los niños afectados por SXF de 6 a I 3 años (Torrioli et al., 2008: 803). También se han comprobado los beneficios de la melatonina para tratar los problemas del sueño que sufren los pacientes afectados por el síndrome (Wirojanan et al., 2009: I45).

En el año 20I4, Hanson y Hagerman llevaron a cabo un estudio retrospectivo con 45 niños con SXF, con edades comprendidas entre I2-50 meses. El análisis encontró que los niños con SXF que recibieron SSRI sertralina tienen una mejoría significativa en el lenguaje en comparación con los niños que no recibieron dicho tratamiento (Hanson y Hagerman, 20I4: I IO). De este estudio surgió un ensayo para el tratamiento del SXF con sertralina en edades comprendidas entre 24 y 68 meses de edad en pacientes con SXF, llevado a cabo por UC Davis MIND Institute (ClinicalTrials.gov: NCToI474746). Se mide el efecto de la sertralina en tres dominios generales: desarrollo de habilidades y lenguaje temprano, habilidades de procesamientos sensorial y sintomatología relacionada con la ansiedad, aprendizaje y el espectro autista (ASD). Los resultados de este ensayo han demostrado mejoras significativas en el lenguaje tras el uso de sertralina en comparación con placebo (Greiss Hess et al., 2016: 619)

La disminución de la proteína FMRP parece estar relacionada también con una alteración de otras sustancias, especialmente las asociadas con la función REDOX celular (Bechara et al., 2009; Davidovic et al., 20I I; El Bekay et al., 2007: 3 I 69; Lima-Cabello et al., 2016; Utine et al., 20I4). Las consecuencias de una alteración del eje hipotálamo-pituitaria-suprarrenal pueden justificar parte del fenotipo conductual del SXF como la inadecuada respuesta al estrés ambiental y psíquico. En modelos animales FMRI-KO se observan exceso de radicales libres dependientes de NADPH-oxidasa que provoca elevación del estrés oxidativo a nivel del sistema nervioso central. Estas alteraciones podrían estar relacionadas con la hiperactividad, el trastorno ansioso y el déficit cognitivo o del lenguaje, pues con la utilización de compuestos reguladores de esta vía fisiopatológica se consigue revertir parcialmente el fenotipo (De Diego Otero et al., 2009: IOI I; Romero-Zerbo et al., 2009: 224; De Diego Otero et al., 20I4).

\section{Ensayos clínicos con antioxidantes en síndrome $X$ frágil}

Los estudios preclínicos publicados en marzo del año 2009 avalan la hipótesis del exceso de radiales libres en cerebro y que un tratamiento antioxidante mejora la sintomatología que manifiesta el ratón Fmr I-KO modelo del síndrome que es comparable a la descrita en pacientes.

Actualmente a pesar de todos los enfoques terapéuticos experimentales, los tratamientos farmacológicos disponibles tienen un efecto limitado sobre los síntomas más frecuentes de los pacientes afectados por el síndrome. En el modelo ratón del síndrome se han demostrado resultados positivos con antioxidantes, lo que ha justificado su ensayo en pacientes y los resultados se han protegido a través de una patente de invención (PCT/ES2OI I/070875) que se ha transferido y actualmente cuenta con la designación de medicamento huérfano por la Agencia Europea del Medicamento, estando actualmente en trámite para su comercialización a finales de 2017. Es importante desarrollar nuevos enfoques terapéuticos experimentales para comprobar la efectividad de compuestos que han resultado positivos en el modelo en ratón del síndrome.

Se han desarrollado varios ensayos terapéuticos experimentales en el síndrome $\mathrm{X}$ frágil en 
pacientes varones y mujeres a partir de 3 años de edad (http://clinicaltrials.gov. NCTor 329770 ), que se han llevado a cabo desde el año 20 Io. Se ha comprobado que la combinación de antioxidantes es efectiva para reducir síntomas como la hiperactividad y la ansiedad que manifiestan los pacientes, mejorando a su vez el lenguaje, así como parámetros cognitivos y la atención (De Diego Otero et al., 2014: 345 ). Actualmente se desarrolla un nuevo ensayo con la combinación de antioxidantes para comprobar su eficacia en niños de I a 8 años de edad, que finalizará en el mes de julio de 2017 (http://clinicaltrials.gov. NCTo2942498). 
Bechara, E. G. et al. (2009): “A novel function for fragile X mental retardation protein in translational activation". PLoS Biol, 7 (г): er6.

Berry-Kravis, E. y Potanos, K. (2004):

"Psychopharmacology in fragile X syndromepresent and future". Ment Retard Dev Disabil Res Rev, Iо (I): 42-48.

Bourgeois, J. A. et al. (2009): "A review of fragile $\mathrm{X}$ premutation disorders: expanding the psychiatric perspective". J Clin Psychiatry, 70 (6): $852-862$.

Brown, V. et al. (200I): "Microarray identification of FMRP-associated brain mRNAs and altered mRNA translational profiles in fragile $\mathrm{X}$ syndrome". Cell, I07 (4): 477-487.

Chen, L. et al. (2003): “The fragile X mental retardation protein binds and regulates a novel class of mRNAs containing $U$ rich target sequences". Neuroscience, I 20 (4): IOO5-IOI7.

Chiurazzi, P. et al. (2003): "Understanding the biological underpinnings of fragile $\mathrm{X}$ syndrome”. Curr Opin Pediatr, I 5 (6): 559-566.

Chiurazzi, P. et al. (I999): "Synergistic effect of histone hyperacetylation and DNA demethylation in the reactivation of the FMRI gene”. Hum Mol Genet, 8 (I 2): 23 I 7-2323.

Davidovic, L. et al. (20I I): “A metabolomic and systems biology perspective on the brain of the fragile X syndrome mouse model". Genome Res, 2I (I2): 2190-2202.

De Diego Otero, Y. et al. (2OI4): “A combination of ascorbic acid and $\alpha$-tocopherol to test the effectiveness and safety in the fragile $\mathrm{X}$ syndrome: study protocol for a phase II, randomized, placebo-controlled trial". Trials, I $5: 345$.

De Diego Otero, Y. et al. (2009): “Alphatocopherol protects against oxidative stress in the fragile X knockout mouse: an experimental therapeutic approach for the Fmri deficiency”. Neuropsychopharmacology, 34 (4): IOI I-IO26.
Dyer-Friedman, J. et al. (2002): "Genetic and environmental influences on the cognitive outcomes of children with fragile X syndrome”. J Am Acad Child Adolesc Psychiatry, 4I (3): 237-244.

El Bekay, R. et al. (2007): "Enhanced markers of oxidative stress, altered antioxidants and NADPH-oxidase activation in brains from Fragile X mental retardation I-deficient mice, a pathological model for Fragile X syndrome”. Eur J Neurosci, 26 (I I): 3 I69-3 I80.

Fernandez-Carvajal, I. et al. (2009): "Screening for expanded alleles of the FMRI gene in blood spots from newborn males in a Spanish population”. J Mol Diagn, I I (4): 324-329.

Fisch, G. S. et al. (1988): "Folic acid treatment of fragile X males: a further study". Am J Med Genet, 30 (I-2): 393-399.

Fung, L. K. y Reiss, A. L. (2016): “Moving Toward Integrative, Multidimensional Research in Modern Psychiatry: Lessons Learned From Fragile X Syndrome”. Biol Psychiatry, 80 (2): IOO-III.

Gatto, C. L. y Broadie, K. (20I0): "Genetic controls balancing excitatory and inhibitory synaptogenesis in neurodevelopmental disorder models". Front Synaptic Neurosci, 2: 4.

Gomis-González, M. et al. (20I6): "Possible Therapeutic Doses of Cannabinoid Type I Receptor Antagonist Reverses Key. Alterations in Fragile X Syndrome Mouse Model”. Genes (Basel), 7 (9).

Greiss Hess, L. et al. (20I6): “A Randomized, Double-Blind, Placebo-Controlled Trial of Low-Dose Sertraline in Young Children With Fragile X Syndrome". J Dev Behav Pediatr, 37 (8): 6I9-628.

Grigsby, J. (2016): “The fragile X mental retardation I gene (FMRI): historical perspective, phenotypes, mechanism, pathology, and epidemiology". Clin Neuropsychol, 30 (6): 8 I $5-833$. 
Hagerman, R. J. et al. (2OI4): “Translating molecular advances in fragile $\mathrm{X}$ syndrome into therapy: a review". J Clin Psychiatry, 75 (4): e294-307.

Hagerman, R. J. (2006): “Lessons from fragile $\mathrm{X}$ regarding neurobiology, autism, and neurodegeneration”. J Dev Behav Pediatr, 27 (I): 63-74.

Hagerman, R. J. y Hagerman, P. J. (2002): Fragile $X$ syndrome. $3^{\text {er }}$ ed. Baltimore: The Johns Hopkins University Press.

Hanson, A. C y Hagerman, R. J. (2OI4): "Serotonin dysregulation in Fragile X Syndrome: implications for treatment". Intractable Rare Dis Res, 3 (4): I IO-I I7.

He, C. X. y Portera-Cailliau, C. (20I3): “The trouble with spines in fragile $\mathrm{X}$ syndrome: density, maturity and plasticity". Neuroscience, 25 I: I 20-I 28.

Iossifov, I. et al. (20I2): "De novo gene disruptions in children on the autistic spectrum". Neuron, 74 (2): $285-299$.

Jacquemont, S. et al. (20I I): "Epigenetic modification of the FMR I gene in fragile $\mathrm{X}$ syndrome is associated with differential response to the $\mathrm{mGluR}_{5}$ antagonist AFQ०56". Sci Transl Med, 3 (64): 64raI.

Leigh, M. J. et al. (2013): "A randomized doubleblind placebo-controlled trial of minocycline in children and adolescents with fragile $\mathrm{x}$ syndrome". J Dev Behav Pediatr, 34 (3): I47I 55 .

Ligsay, A. y Hagerman, R. J. (20I6): "Review of targeted treatments in fragile X syndrome". Intractable Rare Dis Res, 5 (3): I $58-167$.

Lima-Cabello, E. et al. (2016): “An Abnormal Nitric Oxide Metabolism Contributes to Brain Oxidative Stress in the Mouse Model for the Fragile X Syndrome, a Possible Role in Intellectual Disability”. Oxid Med Cell Longev, 2016.

Paribello, C. et al. (2010): "Open-label add-on treatment trial of minocycline in fragile $\mathrm{X}$ syndrome”. BMC Neuron, I0: 9I.
Restivo, L. et al. (2005): "Enriched environment promotes behavioral and morphological recovery in a mouse model for the fragile $\mathrm{X}$ syndrome". Proc Natl Acad Sci U S A, IO2 (32): II $557-\operatorname{II} 562$.

Romero-Zerbo, Y. et al. (2009): "Protective effects of melatonin against oxidative stress in Fmr I knockout mice: a therapeutic research model for the fragile X syndrome". J Pineal Res, 46 (2): 224-234.

Saldarriaga, W. et al. (2OI4): "Fragile X síndrome". Colomb Med (Cali), 45 (4): I90-198.

Sahu, J. K. et al. (20I3): "Effectiveness and safety of donepezil in boys with fragile $\mathrm{x}$ syndrome: a double-blind, randomized, controlled pilot study". J Child Neurol, 28 (5): 570-575.

Sidorov, M. S. et al. (2013): "Fragile X mental retardation protein and synaptic plasticity". Mol Brain, 6: I 5 .

Tang, A. H. y Alger, B. E. (2OI 5 ): "Homer proteinmetabotropic glutamate receptor binding regulates endocannabinoid signaling and affects hyperexcitability in a mouse model of fragile $\mathrm{X}$ syndrome”. J Neurosci. 35 (9):3938-3945.

Tassone, F. et al. (200I): “A majority of fragile $\mathrm{X}$ males with methylated, full mutation alleles have significant levels of FMRI messenger RNA”. J Med Genet. 38 (7): 453-456.

Torrioli, M. G. et al. (2008): “A doubleblind, parallel, multicenter comparison of L-acetylcarnitine with placebo on the attention deficit hyperactivity disorder in fragile $\mathrm{X}$ syndrome boys". Am J Med Genet A, I46 (7): $803-8$ I 2 .

Utine, G. E. et al. (20I4): "Neurochemical evaluation of brain function with $\mathrm{IH}$ magnetic resonance spectroscopy in patients with fragile X syndrome". Am J Med Genet A, I64A (I): 99-105.

Wirojanan, J. et al. (2009): "The efficacy of melatonin for sleep problems in children with autism, fragile $\mathrm{X}$ syndrome, or autism and fragile X syndrome”. J Clin Sleep Med, 5 (2): I $45^{-I} 5$ O. 